\title{
On the Impact of Replica Placement to the Reliability of Distributed Brick Storage Systems
}

\author{
Qiao Lian, Wei Chen, Zheng Zhang \\ Microsoft Research Asia \\ \{t-qiaol,weic, zzhang\}@microsoft.com
}

\begin{abstract}
Data reliability of distributed brick storage systems critically depends on the replica placement policy, and the two governing forces are repair speed and sensitivity to multiple concurrent failures. In this paper, we provide an analytical framework to reason and quantify the impact of replica placement policy to system reliability. The novelty of the framework is its consideration of the bounded network bandwidth for data maintenance. We apply the framework to two popular schemes, namely sequential placement and random placement, and show that both have drawbacks that significantly degrade data reliability. We then propose the stripe placement scheme and find the near-optimal configuration parameter such that it provides much better reliability. We further discuss the possibility of addressing the problem of correlated brick failures in our analytical framework.
\end{abstract}

\section{Introduction}

Storage solution using clustered "smart bricks" connected with LAN is becoming an increasingly attractive alternative to the more expensive SAN (storage-area network) solution. Some of the exemplary systems include Petal [11], NASD [9], GFS [8], FAB [7], Repstore [22], and Boxwood [14]. A smart brick is essentially a stripped down PC with a CPU, memory, network card, and a large disk. For these systems, providing strong data reliability is confronted with new challenges, because inexpensive commodity disks are more prone to permanent failures and failures are far more frequent in large systems. To guard against permanent loss of data, replication is often employed. If some replicas are lost due to disk failures, other replicas are still available and can be used to regenerate new replicas to maintain the same level of reliability.

Replica placement refers to the strategy of placing replicas among the participating bricks. The two widely used replica placement schemes are staggered sequential placement like in chained declustering [10] used by
Petal [12] as well as in many proposals based on DHT (distributed hash table) [4][17], and the totally random placement like in GFS [8]. Mirroring can be viewed as a degenerated special case of sequential placement.

Replica placement can significantly affect the reliability of the system due to two factors. The first is the repair speed: the more bricks participate in the data repair process, subject to the available network bandwidth, the sooner that the reliability level returns. The second is the sensitivity to multiple and concurrent failures: the more permutation choices that the placement generates, the more likely a random failure of several bricks will wipe out some data permanently. These two factors are conflicting in nature. For instance, the random placement has very fast repair speed, but is prone to concurrent failures, whereas the sequential placement is precisely the opposite.

The contributions of the paper are mainly two folds. First, we provide a systematic framework not only to identify, but also to reason and quantify the impact of the replica placement policy to system reliability. In particular, our result points out that under different parameters, random and sequential placement can have vastly different results. Our framework captures the bounded available network bandwidth for data maintenance, something no other models have done.

Second, the insight that we gain from the first result leads us to propose the stripe placement scheme which attempts to achieve the best balance between the two competing forces. While it is difficult to derive a closed form, we do provide the near-optimal configuration parameter verified with simulations. This is the second contribution of this paper.

Moreover, we also sketch a proposal to extend our framework to deal with correlated failures that frequently occur in practice.

The roadmap of the paper is as follows. In Section 2, we discuss the replica placement schemes and the data reliability metric. In Section 3, we present the analytical framework for the reliability study. In Section 4, we apply the framework to the two placement schemes and 
compare the results. In Section 5, we describe the stripe placement scheme, and find the near-optimal parameter for the scheme. We discuss how to deal with correlated failures in Section 6. Related work is summarized in Section 7, and we conclude the paper in Section 8.

\section{Replica Placement and Reliability Metric}

Without loss of generality, we consider an ordered array of $N$ bricks on which replicas are placed. The number of replicas of an object is called the replication degree of the object, and it is denoted by $k$. Replication degree may differ from object to object, but for simplicity we assume all objects have the same $k$. The responsibility of replica placement is to designate the bricks on which the replicas are hosted. All other issues, such as the interface of the system, are orthogonal to the reliability study.

Each individual brick may fail permanently and lose all replicas stored on the brick. In this paper, we equate brick failures with disk failures, since disk failures ultimately cause data loss. When a brick fails, to keep data reliability of the system at the same level, the system needs to automatically regenerate the lost replicas at the remaining bricks. This replica regeneration process is called data repair.

\subsection{Data reliability metric $M T T D L$}

To measure the data reliability of a system, we use the metric $M T T D L$ - mean time to data loss in the entire system. MTTDL indicates, after the system is loaded with data objects, how long on average the system can sustain before it permanently loses the first data object in the system. This is a metric widely used in storage literature, e.g. on RAID storage [2]. ${ }^{1}$

There are two important factors that affect data reliability. The first one is the speed of data repair. Fast data repair means that the lost replicas are likely to be repaired before further brick failures, so it reduces the time window in which concurrent brick failures occur and wipe out all replicas of some object.

The second factor is the likelihood of data loss when concurrent brick failures do occur. When $k$ random bricks fail concurrently in the system, the likelihood that some object whose $k$ replicas are located on the $k$ failed bricks depends on the placement scheme used, as we will discuss shortly. In general, the more likely the concurrent brick failures happen to wipe out all replicas of an object, the less reliable the system is.

\footnotetext{
${ }^{1}$ One may also consider the amount of data loss when it happens. However, when the goal of a storage system is to provide nearly nodata-loss reliability, $M T T D L$ is a more important metric than the amount of data loss. Thus the paper focuses on the analysis of MTTDL.
}

\subsection{Sequential placement}

Sequential placement is simple in nature: one of the brick acts as the lead brick, and the $k$ replicas are placed on the lead brick and its $k-1$ followers. This is what the chained declustering [10] does and is employed in Petal [11]. This scheme is also a common strategy in peer-topeer wide-area storage systems like CFS [4] and PAST [17], in which the lead brick is typically identified by the hash of the object.

With sequential placement, when a brick fails, the $k$ neighboring bricks on each side of the failed brick can participate in brick repair (Figure 1). The limited parallel repair degree leads to slow repair speed, which then negatively affects the data reliability of the system.

However, the restrictive nature of placement reduces the sensitivity to concurrent failures. If and only if $k$ simultaneous failures occur on $k$ consecutive bricks in the ordered array can any object be lost. This is unlikely when $N$ is much larger than $k$. Thus, sequential placement has a low likelihood of data loss when concurrent failures occur, which improves data reliability.

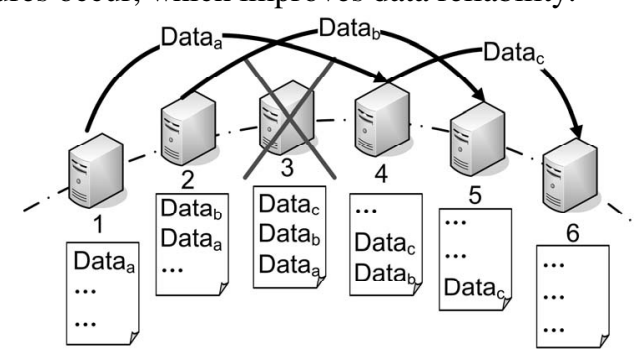

Note: Replication degree is 3 . When brick 3 fails, data repair can be carried out as brick 1 copying Data $_{\mathrm{a}}$ to brick 4 , brick 2 copying Data to brick 5, and brick 4 copying Data $\mathrm{C}_{\mathrm{c}}$ to brick 6 .

\section{Figure 1. Data repair in sequential placement.}

\subsection{Random placement}

In random placement, replicas are placed randomly among the $N$ bricks, and this is used in the work of [8][19]. ${ }^{2}$ The main objective here is to improve the speed of data repair. With random placement, when a brick fails, the replicas on the failed brick can be found on many other bricks, and thus many bricks can participate in data repair in parallel, resulting in faster data repair speed. This is its main advantage over sequential placement in improving data reliability.

However, crashing $k$ random bricks will likely remove all the replicas of some objects with random

\footnotetext{
${ }^{2}$ An indexing scheme is needed for random placement to access all objects in the system. However, indexing is orthogonal to our study of reliability, so we will not discuss it in detail.
} 
placement. In the extreme case when there is a large volume of objects in the system and therefore the actual placement choices have exhausted all possible combinations, any $k$ crashes cause data loss. High sensitivity to multiple and concurrent failures, therefore, is the drawback of the random placement scheme.

It is intuitive to see that neither placement scheme is perfect. As we will reveal in Section 4, under different circumstances their difference can be dramatic.

\section{Analytical Framework}

In this section, we present the analytical framework for the data reliability analysis. The framework shows how to derive MTTDL from known system parameters. This framework can be applied to different object placement schemes, as we will show in the next section.

The novelty of the framework is its consideration of the bounded network bandwidth available for data repair, which directly affects data repair speed.

\subsection{System model for analysis}

We consider a system with $N$ bricks and the replication degrees of all objects are $k$. The average amount of data stored on each brick is $c$. We assume a reasonable amount of free space on each brick for data repair. Brick failures follow an exponential distribution with MTTF (mean time to failure) as its mean. We first assume that each brick fails independently, and later we will consider a model for correlated brick failures. We do not model transient failures of bricks that only affect data availability but not affect data loss. When a brick fails, we assume a new brick is added into the system immediately to keep the system scale at $N$ all the time.

All bricks are connected in a LAN with a root switch. The network provides certain bandwidth for data repair traffic, and the bound of which is given by $B$, which is called the backbone bandwidth. The backbone bandwidth can be viewed as a certain percentage of the bandwidth of the root switch that is allowed for data repair traffic, because in many simple topologies, all (or nearly all) data repair traffic goes through the root switch.

We do not separately consider network failures. A network failure does not cause data loss directly, but it may reduce the data repair speed, and thus affect data reliability. We fold this aspect into the available data repair bandwidth $B$.

\subsection{Analysis}

3.2.1. Introducing $M T T D L_{o b j}$. To conduct the analysis for $M T T D L$, we first introduce an intermediate metric $M T T D L_{o b j}$, which is the mean time to data loss for an arbitrary object. $M T T D L_{o b j}$ measures the data reliability of an individual object stored in the system. If the system contains $m$ objects, and these $m$ objects have independent data loss distributions, then we have $M T T D L=$ $M T T D L_{o b j} / m$. Intuitively, this is because that each object has a data loss rate of $1 / M T T D L_{o b j}$, and when they are considered together in a system, the total data loss rate is $m / M T T D L_{o b j}$ since their individual data loss behaviors are independent.

Of course, when object replicas are placed in the system, their data loss behaviors depend on the failures of the bricks, and thus may not be independent of each other. In particular, if the replicas of two objects are colocated at the same set of bricks, their data loss behaviors are perfectly correlated. In this case, they should be considered as one object instead of two independent objects.

For the above reason, we only consider objects whose replica placements are different. Let $m$ be the total number of different replica placement combinations under a placement scheme. We thus have ${ }^{3}$

$$
M T T D L=M T T D L_{o b j} / m \text {. }
$$

In a system with $N$ bricks, $m$ could be as large as $C(N, k){ }^{4}$

3.2.2. Markov Chain Model. To analyze $M T T D L_{o b j}$, we introduce a Markov model as in Figure 2 to model the evolution of the system with brick failures and data repair. In the model, state $i$ represents the state of the system where exactly $i$ bricks have failed, and the lost replicas on the failed bricks have not been completely repaired.

The arcs in the figure represent the transitions between different states in the system. From state $i$ to $i+1$, one more brick failure occurs, and this could occur on any of the $N-i$ remaining bricks. Thus, the rate of transition is $(N-i) / M T T F$, since the brick failures are independent. Transition from state $i$ to state 0 represents that data repair is completed before a new brick fails. Let $\operatorname{MTTR}(i)$ denote the mean time to repair all the failed replicas in state $i$. Thus the transition rate from state $i$ to state 0 is $1 / \operatorname{MTTR}(i)$. MTTR $(i)$ depends on the size of data to be repaired and the available bandwidth for repair, and it will be determined shortly. In the next section, we will show the advantage of random placement

\footnotetext{
${ }^{3}$ The formula is still an approximation, because different objects may have some but not all their replicas co-located on the same set of bricks and thus their data loss behaviors are correlated. However, such co-locations are dictated by the replication degree $k$. Thus, when the system scale $N$ is much larger than the replication degree $k$, the correlated data loss of objects caused by partial co-locations can be ignored. For sequential placement, we compared the approximation with an accurate analysis and the result shows that the approximation matches with the accurate analysis very well.

${ }^{4} C(x, y)$ denotes the total number of combinations of picking $y$ objects from $x$ objects.
} 
over sequential placement in that random placement has a much smaller $\operatorname{MTTR}(i)$.

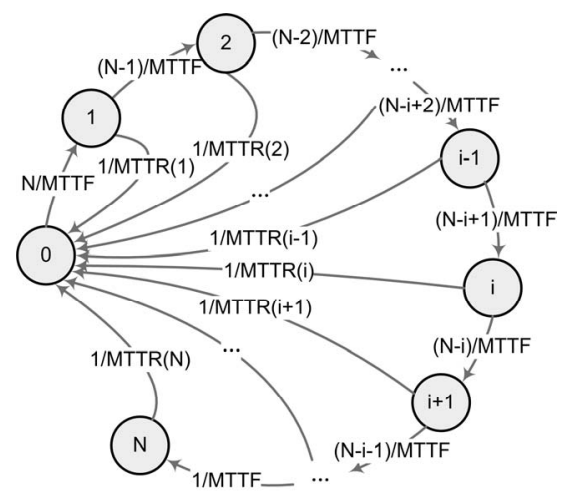

\section{Figure 2. Markov model for reliability analysis}

The Markov model of Figure 2 assumes that there is no transition from state $i$ back to state $j$ with $0<j<i$, which means that data repair for all failed bricks complete at the same time. In reality, data repair for a brick that failed early is likely to be completed early, even without centralized scheduling of data repair. Hence the assumption is a conservative one.

The Markov model of Figure 2 models the evolution of the entire storage system, as opposed to other models (e.g. [20]) that only model the evolution of one object. The reason is that in our environment, data repair traffic is limited by the backbone bandwidth. Thus, more brick failures are likely to slow down data repair because more data repair traffic are sharing the limited bandwidth. Therefore, we have to look at the state of the entire system to determine the speed of data repair.

3.2.3. Deriving $\boldsymbol{M T T D L _ { o b j }}$. This section briefly describes the derivation of $M T T D L_{o b j}$ using the Markov model. The full technical derivation is available in [13]

Let $M T B F(i)$ be the mean time between two consecutive occurrences of state $i$ in the Markov model. Let $L(i)$ be the probability that the object is lost when $i$ bricks fail concurrently. Let $P(i)$ be the probability of the system staying in state $i$. The formula below shows that $M T T D L_{o b j}$ can be calculated from the harmonic sum of mean time between data loss in each individual state $i$.

$$
M T T D L_{o b j}=\left[\sum_{i=k}^{N} M T B F(i)^{-1} \cdot L(i)\right]^{-1} \cdot
$$

Probability $L(i)$ is easily derived from a combinatorial calculation:

$$
L(i)=C(i, k) / C(N, k) .
$$

The derivation of $\operatorname{MTBF}(i)$ is more involved and is detailed in [13]. The idea is to consider a compressed timeline with only time segments when the system is in state $i-1$. In the compressed timeline, when any of the remaining $(N-i+1)$ bricks fails, the $i$-th failure occurs, and the mean time between two consecutive events of the $i$-th failures is $M T T F /(N-i+1)$. Since the system is in state $i-1$ with probability $P(i-1)$, the compressed timeline compresses time period from the original timeline with the ratio $P(i-1)$. Therefore, in the original timeline, we have

$$
\operatorname{MTBF}(i)=\frac{M T T F}{(N-i+1) \cdot P(i-1)} .
$$

The value of $P(i)$ can be computed from the following balance equation for the Markov model:

$$
\left\{\begin{array}{l}
\frac{P(i)}{P(i-1)}=\frac{(N-i+1) / M T T F}{(N-i) / M T T F+1 / \operatorname{MTTR}(i)}, \text { for all } i \geq 1 . \\
\sum P(i)=1
\end{array}\right.
$$

The term that we have not addressed is $\operatorname{MTTR}(i)$, which is an important one discussed separately below.

3.2.4. Deriving MTTR(i). MTTR(i) depends on both the amount of data to repair and repair bandwidth. Let $D(i)$ and $r b(i)$ be the amount of data to repair and the repair bandwidth in state $i$, respectively. Let $T$ be the time to detect a failure in the system (10sec is used for all analysis and simulation). Then

$$
\operatorname{MTTR}(i)=T+D(i) / r b(i) .
$$

The amount of data to repair $D(i)$ depends on both $c$ (the amount of data in the last failed brick), and the amount of the un-repaired data left from the previous state $\operatorname{ur}(i)$.

$$
D(i)=u r(i)+c .
$$

The amount of un-repaired data left from the previous state $\operatorname{ur}(i)$ depends on (a) the total amount of the previous state's data to be repaired $D(i-1)$, (b) the mean time to the next failure in the previous state $m f(i-1)$, and (c) the previous state's repair bandwidth $r b(i-1)$.

$$
u r(i)=\left\{\begin{array}{ccc}
D(i-1)-r b(i-1) \cdot m f(i-1) & (\text { when } & D(i-1)>r b(i-1) \cdot m f(i-1)) \\
0 & (\text { when } & D(i-1) \leq r b(i-1) \cdot m f(i-1))
\end{array}\right.
$$

In state $i-1$, the mean time to next failure $m f(i-1)$ is $\operatorname{MTTF} /(N-i+1)$. So we have

$$
D(i)=\max [D(i-1)-r b(i-1) \cdot \operatorname{MTTF} /(N-i+1), 0]+c
$$

Once $r b(i)$ is known, $D(i)$ can be calculated iteratively by the above formula.

The repair bandwidth $r b(i)$ at state $i$ varies with different placement schemes. In the next section we will determine this value for both sequential placement and random placement.

\section{Comparing Sequential Placement with Random Placement}

The previous section provides the general framework to analyze the data reliability of a distributed brick storage system with a bounded backbone repair bandwidth. 
The two terms undecided in the analysis are: (a) $m$, the possible replica placement combinations in the system; and (b) $r b(i)$, repair bandwidth at state $i$. It is not hard to see that the larger the $m$, the worse the data reliability, while the larger the $r b(i)$, the faster the data repair can be completed and thus the better the data reliability.

Table 1. Key differentiating quantities for sequential placement and random placement

\begin{tabular}{|l|c|c|}
\hline & $m$ & $r b(i)$ \\
\hline Sequential & $N$ & $\min (B, b \cdot k \cdot i / 2)$ \\
\hline Random & $\min (C(N, k),(N \cdot c) /(k \cdot s))$ & $\min (B, b \cdot(N-i) / 2)$ \\
\hline
\end{tabular}

These two terms vary among different placement schemes. In this section, we determine the two terms for both sequential placement and random placement and compute the reliability of the two schemes. Table 1 lists the results and the explanation follows, where $s$ denotes the average object size, and $b$ denotes the brick bandwidth.

\subsection{Sequential placement}

In sequential placement, replicas are restricted to be placed on $k$ consecutive bricks in the ordered array of bricks. This restriction leads to only $N$ possible placement combinations, i.e. $m=N$, which benefits the reliability of the system.

To calculate the repair bandwidth, let $b$ be the maximum bandwidth of a brick. When one brick fails, the replicas on the failed brick need to be regenerated on the $k$ consecutive bricks after the failed brick. So the repair bandwidth could reach $b \cdot k$. However, among these $k$ bricks, at least one brick would also serve as the source for data repair (e.g., brick 4 in Figure 1), bringing the effective bandwidth only to a half, i.e. $b \cdot k / 2$. When $i$ concurrent failures occur, this gives $b \cdot k \cdot i / 2$ (we ignore the situation when multiple failures are within the range of $k$, and this makes the result optimistic). Also, the maximum repair bandwidth cannot exceed the backbone bandwidth $B$. Therefore, we have $r b(i)=\min (B, b \cdot k \cdot i / 2)$.

\subsection{Random placement}

In random placement, replicas are scattered randomly among the bricks in the system. When one brick fails, many other replicas contain the replicas that are lost on the failed brick. So many replicas can act as the source of data repair, vastly bringing up the degree of parallel repair and hence the repair bandwidth.

Quantitatively, the repair bandwidth of the random placement scheme is given by $r b(i)=\min (B, b(N-i) / 2)$. The term $b(N-i) / 2$ means that when a brick fails, half of the remaining bricks contain the replicas need to be generated, and they copy the replicas to the other half of the bricks, which is a good-case scenario but can be closely approximated.

Comparing $r b(i)$ of the two schemes as listed in Table 1, it is clear that the repair bandwidth of the random placement is much higher than that of sequential placement, for relatively small $i$ 's that are mostly relevant to the data reliability. This is the advantage of the random placement scheme.

For the possible placement combination $m$, it depends on the number of objects, which is determined by the size of the object in our environment. Let $s$ denote the average size of an object in the system. When the system has $N$ bricks with the amount of data stored on each brick being $c$ and the replication degree of $k$, the number of objects in the system is $N \cdot c /(k \cdot s)$. Thus, the possible placement combination $m$ is given by $\min (C(N, k), N \cdot c /(k \cdot s)) .{ }^{5}$ This value could be much larger than $N$, the corresponding value of $m$ in sequential placement, especially when the object size is small. This is the major drawback of the random placement scheme that significantly reduces the reliability of the system.

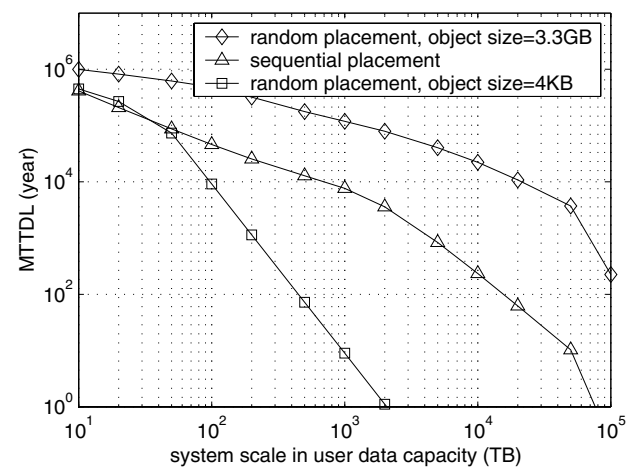

Note: The user data capacity of the system is $c \cdot N / k$. MTTF=1000days, $k=3, \quad B=3 G B / s, \quad b=20 M B / s$, $\mathrm{C}=500 \mathrm{~GB}$. The object size of $4 \mathrm{~KB}$ in random placement is when $m$ reaches $C(N, k)$.

Figure 3. MTTDL of a system vs. the system scale, with sequential placement and random placement.

\subsection{Comparison}

Using the result in Table 1, we can calculate the MTTDL given a set of system configuration parameters. Figure 3 shows the analytical result comparing Sequential placement with random placement.

\footnotetext{
${ }^{5}$ More rigorously, $m$ is the expected number of possible placement combinations for random placement, and it is slightly smaller than $\min (C(N, k), N \cdot c /(k \cdot s))$. We ignore this minor difference in our calculation.
} 
The figure shows several results. First, after the system scale passes a certain point, all schemes essentially stop working: bricks fail so frequently that, with the network bandwidth staying the same, data repair cannot keep up with the brick failures, and the data reliability drops significantly. This means that given a certain backbone bandwidth, the system has a scalability limit.

Second, given the same user capacity, random placement is sensitive to object sizes. The sequential placement scheme is better than random placement for small object size but is worse than that with large object size.

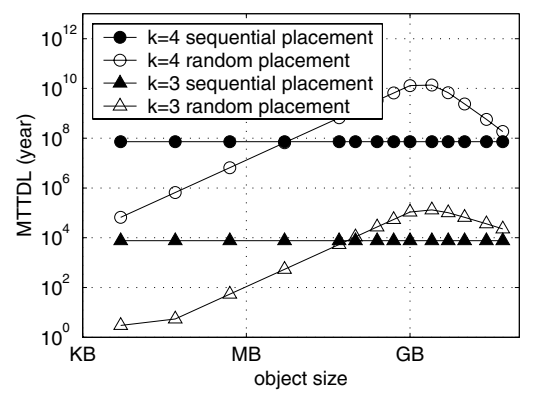

Note: The user capacity of the system is fixed at $1 \mathrm{~PB}=1000 \mathrm{~TB}$. Other system parameters are the same as in Figure 3.

\section{Figure 4. Comparing sequential placement with random placement when varying object size.}

Figure 4 further illustrates the effect of object size on the reliability of the system under the same system scale. In sequential placement, reliability is not affected by object size, because data loss is determined only by the concurrent failures of $k$ consecutive bricks. However, in random placement small object size means a large number of objects, and thus they are more likely to exhaust the possible placement combinations, giving low reliability. When the object size is large, the possible combinations are small, and the benefit of parallel data repair wins over and thus the data reliability is better than sequential placement. But if the object size continues to grow larger, the benefit of parallel repair diminishes and the reliability in random placement returns to the same level as sequential placement.

The figure also compares the result of replication degree of 3 versus 4 . Replication degree of 4 provides close to four orders of magnitude better reliability. In general replication degree of 3 or 4 is suffice to provide enough reliability for most systems.

Overall, no single placement scheme wins in all cases. Random placement gains in fast parallel repair but resulting in too many possible placement combinations when the objects are small, while sequential placement restricts possible placement combinations but is much slower in data repair.

\section{Stripe Placement for Near-Optimal Reli- ability}

Ideally, what we want is the optimal reliability provided by the random placement scheme at its optimal object size, but extending it such that the reliability remains at the same level even for small object sizes. To achieve this effect, we need to group small objects together to make them behave like a large object in terms of placement and repair. Then we can significantly reduce the possible placement combinations that the random placement suffers from, while maintaining a good repair speed. This is the stripe placement scheme we introduce in this section.

\subsection{Stripe placement}

We group small object replicas together to form a large chunk, which is the unit for placement and repair. The sizes of the chunks are the same and will be determined later. The $k$ replicated chunks of the same set of objects form a set called a stripe. A stripe migrates among the bricks in the system with brick failures and data repairs. Figure 5 illustrates the concept of stripe and its repair.

Let stripe number $n_{s}$ be the number of different stripes that can be hosted by one brick. Stripe number determines the degree of parallel data repair. When a brick hosting $n_{s}$ stripes fails, $n_{s}$ different chunks need to be repaired and thus the parallel repair degree is at most $n_{s}$. We will determine the optimal $n_{s}$ shortly. Intuitively, $n_{s}$ should be related to the backbone bandwidth $B$ such that the backbone bandwidth can be fully utilized.

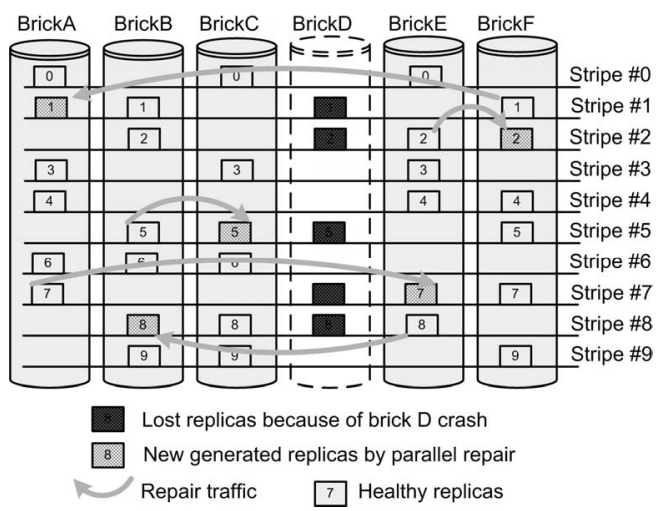

Figure 5. Stripe placement and stripe repair.

We use random placement of chunks and randomly selecting chunk repair sources and destinations to manage the stripes, which is simple in a distributed environment to allow the parallel repair degree to be close 
to $n_{s}$ in spite of brick failures and repairs. In addition to managing random placement of chunks, stripe placement also needs to manage the grouping of objects into stripes. The grouping needs to guarantee that when a new object is added into a stripe, every chunk within the stripe should have enough space to accommodate one replica of the object. To do so, when the stripe is first created, every chunk in the stripe should pre-allocate enough space for the entire chunk. Other management details are omitted here.

\subsection{Finding the optimal $\boldsymbol{n}_{\boldsymbol{s}}$}

We now need to find the optimal $n_{s}$ so that the reliability of the system is the highest. To do so, we use the analytical framework to calculate the reliability of the system for different $n_{s}$, and then locate the $n_{s}$ that provides the optimal reliability.

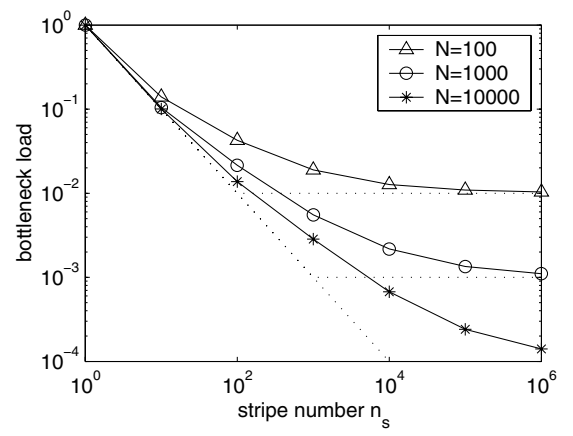

Note: The dotted lines represent the ideal cases where there is no bottleneck brick. The Monte-Carlo simulation is basically throwing $n_{s}$ balls (chunks) into $\mathrm{N}-1$ slots (remaining bricks) and look for the slot with the largest number of balls.

\section{Figure 6. Bottleneck load vs. the stripe number}

First, for the number of possible placement combinations, we have $m=n_{s} \cdot N / k$, since each brick hosts $n_{s}$ stripes and each stripe is hosted by $k$ bricks. Second, for the repair bandwidth, ideally all $n_{s}$ chunks on the failed brick will be repaired by $n_{s}$ different pairs of sources and destinations, in which case the repair bandwidth is $r b(i)=\min \left(B, b \cdot n_{s}\right)$. However, with random chunk placement the repair load may not be even: Some bricks may have more chunks to repair than others. The bottleneck brick is the one with the highest repair load. Let $H$ be the number of stripes to be repaired in the bottleneck brick. We call $H / n_{s}$ the bottleneck load, and denote it as $l_{b}$. We use a Monte-Carlo simulation to calculate $l_{b}$, and the result is shown in Figure 6. The key result is that when $n_{s}$ is close to $N$, the bottleneck load could be one order of magnitude larger than $1 / n_{s}$, the load in the ideal case.
Given the bottleneck load, we can have the repair time based on the bottleneck load, which is $c \cdot l_{b} / b$, where $c$ is the amount of data in a brick, and $b$ is the brick bandwidth. Combining this with the repair time calculation in the framework (formula (2)), we have the following repair time formula for the stripe placement scheme:

$$
\operatorname{MTTR}(i)=T+\max \left[D(i) / \min \left(B, b \cdot n_{s}\right), c \cdot l_{b} / b\right]
$$

Plugging in the above formula in the analytical framework, together with $m=n_{s} \cdot N / k$, we can compute the reliability of the stripe placement scheme.

The results of the analysis are shown here as a series of contour plots (Figure 7). In Figure 7(a), we see that when the stripe number increases while fixing the backbone bandwidth (walking up vertically through the contour), MTTDL increases first because the repair bandwidth increases when more bricks are involved in repair. After reaching a peak $M T T D L$ drops, because the repair bandwidth is restricted by the backbone bandwidth so that repair speed has no further improvement, but the number of possible placement combinations continues to increase as $n_{s}$ increases. So for each backbone bandwidth value, there is an optimal stripe number to give the best MTTDL. From the plot, we can see that the best $M T T D L$ values are located along the ridge in the contour plot, and along this ridge, the stripe number $n_{s}$ increases proportionally to the backbone bandwidth.

Figure 7(b) shows the contour plot of MTTDL with different brick bandwidth and stripe numbers. Similar to Figure 7(a), we can see that the optimal MTTDL values are also located on the ridge of the plot. However, in this case, the optimal stripe number is reverseproportional to the brick bandwidth. Figure 7(c) shows that the optimal stripes number does not rely on the system scale.

Therefore, from the three plots, the conclusion we reach is that the optimal stripe number $n_{s}$ should be proportional to the backbone bandwidth $B$, reverseproportional to the brick bandwidth $b$, and not related to the system scale $N$. Based on this result, we propose that the optimal stripe number $n_{s}$ can be given by $B / b$. In the above three plots, the dash-dotted lines correspond to the stripe numbers with $B / b$. We can see that they are all very close to the ridges, i.e., the best $M T T D L$ values.

The formula $n_{s}=B / b$ is a "guideline" formula. Given a set of system parameters, one can use our analytical framework and some numerical method to find the true optimal $n_{s}$, and the result may be a little different from $B / b$. However, from our analysis, we see that $B / b$ provides near-optimal system reliability. Thus, the optimal chunk size is $c \cdot b / B$ as a function of disk bandwidth, backbone bandwidth and disk capacity. 


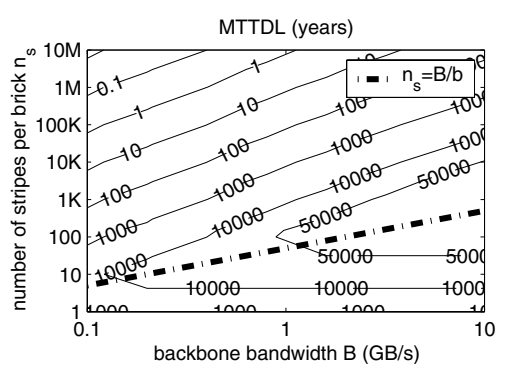

(a) Vary with backbone bandwidth. MTTF $=1000$ days, $b=20 \mathrm{MB} / \mathrm{s}$, $\mathrm{C}=500 \mathrm{~GB}, \mathrm{k}=3$, user capacity $=1 \mathrm{~PB}$.

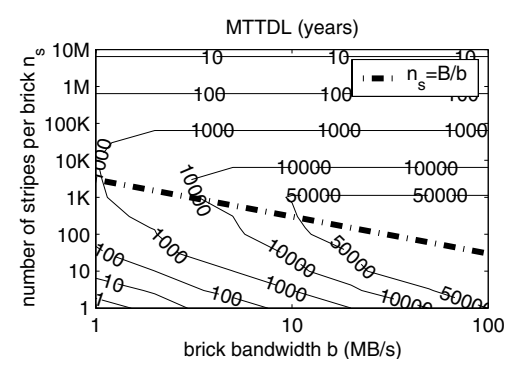

(b) Vary with brick bandwidth. MTTF $=1000$ days, $B=3 \mathrm{~GB} / \mathrm{s}$, $\mathrm{C}=500 \mathrm{~GB}, \mathrm{k}=3$, user capacity $=1 \mathrm{~PB}$.

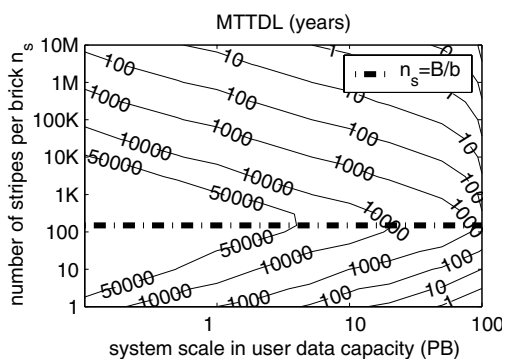

(c) Vary with system scale. MTTF $=1000$ days, $B=3 \mathrm{~GB} / \mathrm{s}$, $\mathrm{b}=20 \mathrm{MB} / \mathrm{s}, \mathrm{c}=500 \mathrm{~GB}, \mathrm{k}=3$.

Figure 7. Contour plot for system MTTDL

The recommendation of $n_{s}=B / b$ allows a simple and intuitive explanation. In the ideal situation, $n_{s}$ pairs of sources and destinations participate in data repair in parallel, and each pair can have maximum bandwidth of $b$. If $n_{s}=B / b$, then the overall repair bandwidth is $n_{s} b=B$, which means the repair exactly saturates the available network bandwidth. This is the best that one can expect. Therefore $n_{s}=B / b$ provides near optimal reliability.

With $n_{s}=B / b$, stripe placement provides much better reliability than sequential placement and random placement. As a numerical example, with our typical setting of $\mathrm{B}=3 \mathrm{~GB} / \mathrm{s}, \mathrm{b}=20 \mathrm{MB} / \mathrm{s}, k=3, c=500 \mathrm{~GB}$, and total user capacity of $1 \mathrm{~PB}$, our optimal stripe placement achieves an $M T T D L$ of $9.41 \cdot 10^{4}$ years, and this does not vary with object size. In contrast, the sequential placement has an MTTDL of $7.66 \cdot 10^{3}$ years, and the random placement has $M T T D L$ values worse than sequential placement when the average object size is less than a few tens of megabytes, and its $M T T D L$ is only getting close to the optimal value when the average object size is in the gigabyte range, as shown in Figure 4.

Furthermore, Figure 7 shows that a reasonable range around the optimal stripe number (e.g. within an order of magnitude change) still permits good reliability that is close to the optimal MTTDL. This means that the guideline of $B / b$ is reasonably robust and may still be applicable even when the network or disks are upgraded over the years.

\subsection{Simulation results}

To verify our analytical results, we run simulations of a brick storage system to see if the reliability matches with the theoretical prediction and if stripe placement indeed provides better reliability.

The simulation is done in an event-driven model. There are two kinds of events pushing the virtual time forward. One is brick failure events, which are triggered by exponentially distributed and independent brick failures. The other is stripe repair finish events, which are triggered at the time when the first stripe repair session is finished. When any event occurs, simulator recalculates the repair bandwidth for every remaining stripe repair session based on the brick and backbone bandwidth constraints. All stripes have the same weight when competing for bandwidth.

Figure 8 shows the results of both the simulation and the theoretical analysis on stripe placement and sequential placement. The main results are: (a) stripe placement is better than sequential placement, and (b) for stripe placement, simulation results match well with theoretical results, while for sequential placement, simulation results show much lower MTTDL because the theoretical analysis on sequential placement is optimistic.

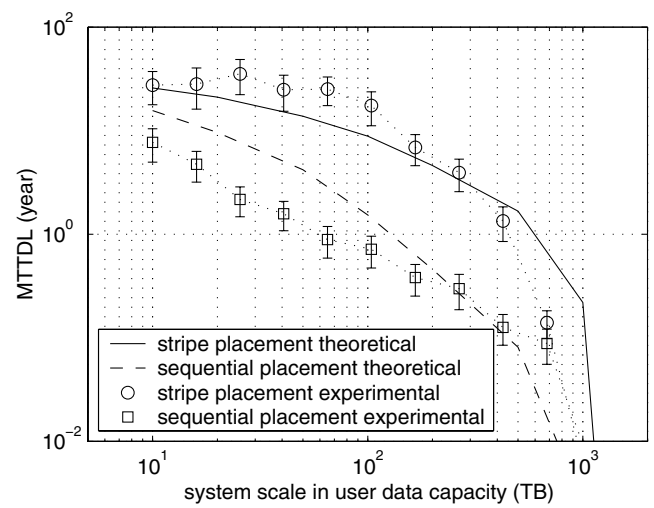

Note: Each simulation result is computed as the average from 50 simulations, with $99 \%$ confidence interval shown in the figure. $M T T F=30$ days, $B=1 \mathrm{~GB} / \mathrm{s}, \mathrm{b}=20 \mathrm{MB} / \mathrm{s}, \mathrm{c}=500 \mathrm{~GB}, \mathrm{k}=3$. The artificially small value of MTTF is to shorten the MTTDL of the system so that the simulations can end within a reasonable amount of time.

\section{Figure 8. Simulation result on MTTDL compar- ing with the theoretical analysis.}

For pure random placement, the simulation with large object size is relatively close to sequential placement, while the simulation with small object size is not 
done because the bandwidth allocation calculation in this case is prohibitively slow.

\section{Discussion on Correlated Failures}

So far, our analysis assumes that brick failures are independent of each other. However, correlated failures are frequently observed in practice, because bricks are usually from the same manufacturer, and they are operated under the same environment with similar access patterns. The primary difficulty here is how to model correlated failures. In our full report [13], we provide a simple proposal that adapts our framework to deal with correlated failures. The proposal is still preliminary and requires further validation. In this section, we sketch our approach of dealing with correlated failures.

Our assumption is that correlated failures do not change the MTTF of each individual brick, because MTTF is measured in a typical operational environment. However, when looking at a large number of bricks, their failures are correlated in the sense that when one brick fails, more bricks are likely to fail soon. Thus, the overall failure behavior is the clustering of failures events separated by a quiet period with few failures.

Accordingly, we convert the failure behavior of the independent failure model into the behavior of correlated failure model by compressing failure events in the independent model into a short period of time, which is followed by a quiet period with no failures. Thus, in the correlated failure model, the failure events are more clustered, but the overall failure rates remain the same, which means that individual MTTF remains the same.

The computation of MTTDL still uses the framework developed for independent failures but it is on the compressed timeline for correlated failures. The result is then stretched back to the original timeline.

This approach allows us to analyze data reliability in the environment that exhibits clustered and correlated failures. However, its correctness and usefulness requires further validation in practice.

\section{Related Work}

Reliability is one of the key aspects of storage systems, and it has been studied extensively, especially for disk arrays like RAID systems [15]. Reliability studies on disk arrays investigate the impact of disk organizations on MTTDL, and typically use Markov models to study the system with independent and exponentially distributed disk failures, e.g. [2] [20]. Our work can be viewed as an extension of these studies into distributed brick storage systems as a generic object store. The important new addition to previous models is the consideration of bounded network bandwidth to data repair.
Many studies [12][1][8][22][14][18] are on similar brick storage systems, but their focuses are not on the systematic study of data reliability. Many of them do address replica placement issues for various reasons. The sequential placement strategy has been widely used. For example, Petal [12] uses chained declustering [10] mainly as a way to improve load balancing, and many P2P systems such as PAST [17] and CFS [4] use it to simplify management. GFS [8] uses random placement to improve data repair performance, but it does not provide a study on the resulting reliability of the system. In our paper, we study the tradeoff to reliability between sequential and random placement.

Several works have investigated the impact of replica placement on availability, not reliability. In Farsite [1], Douceur and Wattenhofer studies dynamic replica placement strategies that improve the overall availability of files [5][6]. In [18][19], van Renesse and Schneider study DHT-based placement (which is categorized as sequential placement in this paper) and random placement and their effects on the availability of a distributed storage system. They use simulation methods and do not consider the effect of available network bandwidth on data reliability.

We have proposed the stripe placement policy with near optimal configuration parameters. Grouping objects is not a new concept. The 64MB chunk size in GFS is based on its workload and read/write performance considerations, while the work in [18] groups a set of objects into volumes but it is not clear how the number of volumes is determined. This study points out that, from a reliability point of view, the chunk size should be a function of available bandwidth, disk bandwidth and disk capacity.

Many coding schemes, in particular Reed-Solomon coding [16], are used in RAID-like storage systems. We do not incorporate such coding schemes into the analysis of reliability, partly because brick storage systems can typically afford more bricks to support simple replication and avoid the complexity and performance penalty associated with the coding schemes.

Some previous work (e.g. [3]) studies the effect of correlated failures, but we do not find a generic model that can facilitate the analysis of system reliability. Our model is simple enough to incorporate into our framework for reliability study, but its effectiveness needs to be further validated in practice.

\section{Concluding Remarks}

With an analytical framework that incorporates available network bandwidth consideration, we study the reliability of distributed storage systems with different replica placement schemes. We show that both sequential placement and pure random placement have 
their drawbacks and propose the stripe placement scheme to achieve near-optimal reliability.

We wish that this study could serve as a guideline for system designers and administrators to determine a number of system parameters when building such brick storage systems, including the root switch bandwidth, the stripe number, the replication degree, etc. We believe that, even though our calculations are based on an idealized framework, the recommendations derived (e.g. using $B / b$ as the stripe number) are applicable to many practical situations, because a reasonable range of values around the computed values provide the same level of reliability as shown by our results. Furthermore, after removing the backbone bandwidth constraint, the framework should be able to adapt to wide-area peer-topeer storage settings.

As the next step, we plan to implement stripe placement in BitVault [21], a data retention platform built with a large number of storage bricks. Future research also includes the study on the reliability with heterogeneous brick components, and the reliability of placement schemes that are aware of network topologies.

\section{References}

[1] A. Adya, W. J. Bolosky, M. Castro, et al, "FARSITE: Federated, Available, and Reliable Storage for an Incompletely Trusted Environment", in Proc. of the 5th OSDI, December 2002.

[2] W. A. Burkhard, J. Menon, "Disk Array Storage System Reliability", in Proc. of Symposium on Fault-Tolerant Computing, 1993.

[3] P. Corbett, B. English, A. Goel, et.al., "Row-diagonal parity for double disk failure correction", in Proc. of $3^{\text {rd }}$ Usenix conference on File and Storage Technologies (FAST'04), April 2004.

[4] F. Dabek, M. F. Kaashoek, D. Karger, et al, "Wide-area cooperative storage with CFS", Proc. of the $18^{\text {th }}$ ACM Symposium on Operating System Principles, 2001.

[5] J. R. Douceur and R. P. Wattenhofer. "Competitive hillclimbing strategies for replica placement in a distributed file system", in Proc. of the $15^{\text {th }}$ Symp. on Distributed Computing. Oct. 2001.

[6] J. R. Douceur and R. P. Wattenhofer, "Optimizing file availability in a secure serverless distributed file system", in Proc. Of the $20^{\text {th }}$ Symp. on Reliable Distributed Systems. IEEE, 2001

[7] S. Frolund, A. Merchant, Y. Saito, et al, "FAB: enterprise storage systems on a shoestring", HOTOS'03.

[8] S. Ghemawat, H. Gobioff, and S.-T. Leung, "The Google File System", in Proc. of the $19^{\text {th }}$ ACM Symposium on Operating System Principles, Oct. 2003

[9] G. A. Gibson, D. F. Nagle, K. Amiri, K., et al. "A CostEffective, High-Bandwidth Storage Architecture", ASPLOS, October, 1998.

[10] H-I. Hsiao and D. J. DeWitt, "Chained declustering: a new availability strategy for multiprocessor database machines", Technical Report CS TR 854, University of Wisconsin, Madison, June, 1989.

[11] J. Kubiatowicz, D. Bindel, Y. Chen, et al, "OceanStore: An Architecture for Global-Scale Persistent Storage", ASPLOS 2000.

[12] E. K. Lee and C. A. Thekkath, "Petal: Distributed Virtual Disks", ASPLOS 1996.

[13] Q. Lian, W. Chen, and Z. Zhang, "On the impact of replica placement to the reliability of distributed storage systems", Microsoft Research Technical Report, to appear.

[14] J. MacCormick, N. Murphy, M, Najork, et.al, "Boxwood: Abstractions as the Foundations for Storage Infrastructure", In Proc. of OSDI'04, Dec. 2004.

[15] D. A. Patterson, G. Gibson, R. H. Katz, "A case for redundant arrays of inexpensive disks (RAID)", In Proc. of the 1988 ACM SIGMOD international conference on Management of data, 109 - 116, 1988.

[16] J. S. Plank. A tutorial on Reed-Solomon coding for fault-tolerance in RAID-like systems. Software --- Practice and Experiene, 27(9):995-1012, Sept. 1997.

[17] A. Rowstron and P. Druschel, "Storage management and caching in PAST, a large scale, persistent peer-to-peer storage utility. In Proc. Of the $18^{\text {th }}$ ACM Symp. On Operating Systems Principles, October 2001.

[18] R. van Renesse, F. B. Schneider, "Chain replication for Supporting High Throughout and Availability", In Proc. of OSDI'04, Dec. 2004.

[19] R. van Renesse, "Efficient Reliable Internet Storage", Workshop on Dependable Distributed Data Management. Oct., 2004.

[20] Q. Xin, E. L. Miller, D. D. E. Long, S. A. Brandt, T. Schwarz, W. Litwin, "Reliability Mechanisms for Very Large Storage Systems", in Proc. of 20th IEEE/11th NASA Goddard Conference on Mass Storage Systems \& Technologies, Apr., 2003

[21] Z. Zhang, Q. Lian, S. D. Lin, et al, "BitVault: A highly reliable distributed data retention platform", submitted for publication.

[22] Z. Zhang, S. D. Lin, Q. Lian, et al, "RepStore: A SelfManaging and Self-Tuning Storage Backend with SmartBricks", In Proc. Of the first IEEE International Conference on Autonomic Computing, May 2004. 